\title{
Letter
}

\section{Dear Feminist Review}

I am writing to express my concern at the unpleasant tone of Janet Rachel's review of Gender \& Technology in the Making by Cynthia Cockburn and Susan Ormrod. I believe that as a matter of developing a tolerant exchange between feminist scholars it should be unacceptable to conclude a review with the sentence: 'It is to these skills that we must attend if we are to be anything other than technically incompetent badge wearing feminists'.

It happens that as a feminist sociologist of science, and to some extent technology, I think the Cockburn and Ormrod book is stunningly good, and that I suspect an enormous number of feminists and others will get great intellectual pleasure from reading it. But the difference in our evaluation of the text is a matter of judgement. For example, in my view it is sheer dualistic rot to suggest, as Rachel does, that it is impossible 'to claim both Harding and Haraway as equally relevant to the study'. Any careful reading of the exchanges between these two would see the mutually shaping conversation developing between them. But the proof of the pudding is in the eating, not in a sour review. Go read.

Yours sincerely,

\section{Hilary Rose,}

Institute of Education,

18 Woburn Square, London WC1H ONS 